\title{
Tanshinone IIA induces apoptosis of ovarian cancer cells in vitro and in vivo through attenuation of PI3K/AKT/JNK signaling pathways
}

\author{
XIAN ZHANG ${ }^{1 *}$, YONG ZHOU ${ }^{2 *}$ and YING-ER GU ${ }^{1}$ \\ Departments of ${ }^{1}$ Chinese Integrative Medicine and ${ }^{2}$ Gynecologic Oncology, Women's Hospital, \\ School of Medicine, Zhejiang University, Hangzhou, Zhejiang 310006, P.R. China \\ Received May 24, 2017; Accepted January 18, 2018
}

DOI: $10.3892 / \mathrm{ol} .2018 .9744$

\begin{abstract}
Ovarian cancer is one of the most common gynecological tumors and is the second most common cause of gynecological cancer-associated mortality worldwide. Tanshinone IIA (Tan-IIA) possesses anticancer activities through inducing the apoptosis of tumor cells. The purpose of the present study was to analyze the ability of Tan-IIA to induce apoptosis of human ovarian cancer cells in vitro and in vivo, and to examine the potential mechanism underlying its activity. Western blot analysis, immunohistochemistry and flow cytometry were used to analyze the therapeutic effects of Tan-IIA on ovarian cancer. It was demonstrated that Tan-IIA significantly inhibited the growth and aggressiveness of human ovarian cancer cells. Tan-IIA significantly increased the apoptosis of human ovarian cancer cells through cleavage activation of caspases-3, caspase- 8 and caspases-9. In addition, Tan-IIA treatment decreased the expression of mitochondrial-protective B-cell lymphoma 2-like protein 2 (Bcl-w) and myeloid cell leukemia-1 long (Mcl-1L) in ovarian cancer cells. Tan-IIA also reduced the expression of phosphoinositide 3-kinase (PI3K), AKT and c-Jun N-terminal kinase (JNK) in human ovarian cancer cells. A specific PI3K inhibitor (LY294002) enhanced the Tan-IIA-inhibited expression of AKT and JNK. The overexpression of PI3K negated the Tan-IIA-inhibited expression of AKT and JNK, and eliminated the Tan-IIA-induced apoptosis of human ovarian cancer cells. Additionally, the in vivo assay showed that Tan-IIA treatment inhibited the growth of ovarian cancer through increasing the apoptosis of tumor cells.
\end{abstract}

Correspondence to: Professor Ying-Er Gu, Department of Chinese Integrative Medicine, Women's Hospital, School of Medicine, Zhejiang University, 1 Xueshi Road, Hangzhou, Zhejiang 310006, P.R. China

E-mail: zjgye@zju.edu.cn

${ }^{*}$ Contributed equally

Key words: tanshinone IIA, ovarian cancer, apoptosis, phosphoinositide 3-kinase/AKT/c-Jun N-terminal kinase
In conclusion, these findings suggested that the induction of apoptosis by Tan-IIA involves the PI3K/AKT/JNK signaling pathways in ovarian cancer.

\section{Introduction}

Ovarian cancer is one of the most common types of tumor in women worldwide, and exhibits increasing morbidity and mortality rates $(1,2)$. Despite medical advances in surgical techniques, radiation, chemotherapy and gene target therapy, the five-year survival rate of patients with ovarian cancer remains poor $(3,4)$. Resistance to anti-ovarian cancer therapies is a major issue in patients with recurrent ovarian cancer $(5,6)$. Currently, apoptotic resistance is an important factor in chemotherapy-managed metastatic ovarian cancer in patients $(7,8)$. The biological relevance of Chinese medicinal therapy in the progression of breast cancer is evidenced by the increasing immunosuppression, which has demonstrated their capacities to induce apoptosis and inhibit tumor aggressiveness $(9,10)$. Therefore, the development of Chinese medicine monomers that overcome apoptotic resistance has been the focus of investigations in the treatment of cancer.

Tanshinone IIA (Tan-IIA), a traditional Chinese medicine extracted from Danshen, has been clinically used for the treatment of human cancer (11-13). In previous years, experimental investigation of the anticancer mechanism of Tan-IIA against human breast cancer has identified the downregulation of multiple genes, including P53 and B-cell lymphoma 2 (bcl-2), which are involved in cell cycle regulation, cell proliferation, apoptosis and DNA synthesis (14). The pharmacological and therapeutic properties of Tan-IIA in inducing the apoptosis of human cancer cells have attracted increased interest through the induction of reactive oxygen species and decreasing the mitochondrial membrane potential $(15,16)$. A previous study indicated that Tan-IIA can inhibit the growth of lung cancer H146 cells by upregulating pro-apoptotic gene expression and decreasing mitochondrial membrane potential (15). Of note, a previous study indicated that Tan-IIA induced apoptosis via p38 mitogen-activated protein kinase (MAPK), and the downregulation of excision repair cross-complementing 1 and lung-resistance protein in cisplatin-resistant ovarian cancer cells (17). Therefore, Tan-IIA is widely used for the treatment of ovarian cancer. 
On these premises, the present study developed a series of experimental strategies to identify the role of Tan-IIA in ovarian cancer growth and examine the possible mechanism of its activity. It was hypothesized that Tan-IIA induces the apoptosis of ovarian cancer cells through attenuation of the phosphoinositide 3-kinase (PI3K)/AKT/c-Jun N-terminal kinase (JNK) signaling pathways.

\section{Materials and methods}

Cell culture. The A2780 ovarian cancer cells were purchased from American Type Culture Collection (Rockville, MD, USA). All cells were cultured in Dulbecco's modified Eagle's medium (DMEM; Invitrogen; Thermo Fisher Scientific, Inc., Waltham, MA, USA) with $10 \%$ fetal bovine serum (FBS; Invitrogen; Thermo Fisher Scientific, Inc.) and $1 \%$ penicillin/streptomycin (Invitrogen; Thermo Fisher Scientific, Inc.) in a $37^{\circ} \mathrm{C}$ humidified atmosphere of $5 \% \mathrm{CO}_{2}$.

MTT cytotoxicity assays. The A2780 cells $\left(1 \times 10^{3}\right)$ were incubated with Tan-IIA $(50,100,150,200$ and $250 \mu \mathrm{M})$ from Herbasin Co., Ltd. Shenyang, China (purity $>99.2 \%$ ) in 96-well plates for $24 \mathrm{~h}$, in triplicate for each condition, with phosphate buffer solution (PBS) added instead of the Tan-IIA as a control. Subsequently, $20 \mu \mathrm{l}$ of MTT $(5 \mathrm{mg} / \mathrm{ml})$ in PBS solution was added to each well, and the plate was incubated for another $4 \mathrm{~h}$. The majority of the medium was removed and $100 \mu \mathrm{l}$ of dimethyl sulfoxide was added to the wells to solubilize the crystals. The optical density (OD) was measured using a Bio-Rad ELISA reader (Bio-Rad Laboratories, Inc., Hercules, CA, USA) at a wavelength of $490 \mathrm{~nm}$.

Overexpression of PI3K. The A2780 cells $\left(1 \times 10^{5}\right)$ were cultured in a six-well plate until $85 \%$ confluence, following which the medium was removed from the culture plate followed by three PBS washes. The A2780 cells were transfected with pLentivirus-PI3K (pPI3K) using Lipofectamine 2000 (Sigma-Aldrich; Merck Millipore, Darmstadt, Germany). The PI3K-overexpressing A2780 cells were then treated with Tan-IIA $(150 \mu \mathrm{M})$ and/or PI3K inhibitor $(50 \mathrm{mg} / \mathrm{ml}$; LY294002; Qiagen, Inc., Gaithersburg, MD, USA) for $12 \mathrm{~h}$ at $37^{\circ} \mathrm{C}$ to analyze the protein expression levels, determined by western blot analysis.

Cell invasion and migration assays. The A2780 cells $\left(1 \times 10^{5}\right)$ were transfected with pPI3K, PI3K inhibitor and/or Tan-IIA $(150 \mu \mathrm{M})$. For the migration assay, the A2780 cells were seeded onto a Matrigel Invasion Chamber (BD Biosciences, San Jose, CA, USA) for $24 \mathrm{~h}$ at $37^{\circ} \mathrm{C}$. For the invasion assay, the A2780 cells were suspended as a density of $1 \times 10^{5}$ in $500 \mu \mathrm{l}$ serum-free DMEM. The cells were then added to the top of the BD BioCoat Matrigel Invasion Chamber (BD Biosciences) for $24 \mathrm{~h}$ according to the manufacturer's protocol. The cells invaded through the Matrigel were fixed in methanol $(25 \%)$ at 24-h post-treatment. The cells were then stained with $0.1 \%$ crystal violet in $25 \%$ methanol, followed by washing in cold 1X PBS. The tumor cell invasion and migration were counted in at least three randomly selected stained fields of every membrane under a microscope (IX71; Olympus Corporation, Tokyo, Japan) at 10X magnification.
Flow cytometric analysis. The A2780 cells $\left(1 \times 10^{5}\right)$ were cultured in a six-well plate until $90 \%$ confluence was reached. Apoptosis was assessed following incubation of the A2780 cells with Tan-IIA $(150 \mu \mathrm{M})$ and/or PI3K inhibitor $(50 \mathrm{mg} / \mathrm{ml})$ for $48 \mathrm{~h}$. The A2780 cells were trypsinized and collected following incubation. The cells were then washed in cold PBS, adjusted to $1 \times 10^{6}$ cells/ml with PBS, labeled with Annexin V-FITC and PI using an Annexin V-FITC kit (BD Biosciences), and analyzed with a FACScan flow cytometer (BD Biosciences).

Western blot analysis. The A2780 cells $\left(1 \times 10^{8}\right)$ were homogenized in lysate buffer containing protease-inhibitor and were centrifuged at $6,000 \mathrm{x} \mathrm{g}$ at $4^{\circ} \mathrm{C}$ for $10 \mathrm{~min}$. Protein concentration was measured by a BCA protein assay kit (Thermo Fisher Scientific, Inc.). Protein extracts $(10 \mu \mathrm{g})$ were electrophoresed on $8-12 \%$ denaturing gel and transferred to a polyvinylidene fluoride membrane (GE Healthcare, Chicago, IL, USA) for western blotting analysis. as previously described (18). For western blot analysis, the following rabbit anti-rat primary antibodies were used: JNK (1:1,000, cat. no. ab124956, Abcam Cambridge, MA, USA), PI3K (1:500, cat. no. ab86714, Abcam), AKT (1:500, cat. no. ab64148, Abcam), phosphorylated (p)JNK (1:1,000, cat. no. ab47337, Abcam), pAKT (1:500, cat. no. ab8932, Abcam), caspase-3 (1:1,000, cat. no. ab2171, Abcam), caspase-8 (1:1,000, cat. no. ab25901, Abcam), caspase-9 (1:1,000, cat. no. ab52298, Abcam), Bcl-2-like protein 2 (Bcl-w; 1:1,000, cat. no. ab38629, Abcam), myeloid cell leukemia-1 long (Mcl-1L; 1:1,000, cat. no. ab32087, Abcam) and $\beta$-actin $\left(1: 2,000\right.$, cat. no. ab8226, Abcam) for $12 \mathrm{~h}$ at $4{ }^{\circ} \mathrm{C}$. The antibodies were added following blocking $(5 \%$ skimmed milk) for $1 \mathrm{~h} 37^{\circ} \mathrm{C}$ and were subsequently incubated with horseradish peroxidase (HRP)-conjugated goat anti-rabbit IgG monoclonal antibody (cat. no. PV-6001, ZSGB-BIO, Beijing, China) for $12 \mathrm{~h}$ at $4^{\circ} \mathrm{C}$. A Ventana Benchmark automated staining system was used for visualizing protein expression in tumor tissues (Olympus BX51; Olympus Corporation).

Animal experiments. A total of 100 female Sprague-Dawley rats (8-week old, 300-320 g body weight) were purchased from Slack Co., Ltd. (Shanghai, China). All animals were housed in a temperature-controlled facility at $23 \pm 1^{\circ} \mathrm{C}$ and relative humidity of $50 \pm 5 \%$ with a 12-h light/dark cycle. All rats had ad libitum access to food and water. The rats were subcutaneously implanted with A2780 tumor cells $\left(1 \times 10^{7}\right)$. The rats received either Tan-IIA treatment $(10 \mathrm{mg} / \mathrm{kg})$ via gavage or treatment with the same volume of PBS control ( $n=10$ in each group, once a day for 1 week) on day 5 . The tumor volume was calculated as follows: Volume $\left.=(\mathrm{D} \mathrm{x} \mathrm{d})^{2}\right) / 2$, where $\mathrm{D}$ represents the maximal diameter and $d$ represents the minimal diameter. The rats were sacrificed on day 30 following a total of seven treatments.

Immunohistochemistry. Tumors from the xenograph rats were fixed using formaldehyde (10\%), followed by embedding in paraffin. The tumor tissues were fabricated into 4- $\mu$ m-thick tumor sections. Antigen retrieval was performed on the tumor sections and the sections were incubated for $12 \mathrm{~h}$ at $37^{\circ} \mathrm{C}$ with the following primary antibodies: JNK (1:1,000, cat. no. ab124956, Abcam), PI3K (1:500, cat. no. ab86714, Abcam), AKT (1:500, cat. no. ab64148, Abcam) The sections were washed three times with PBS 
A

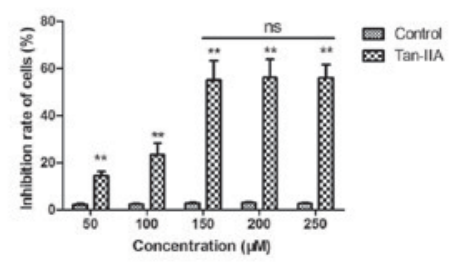

B
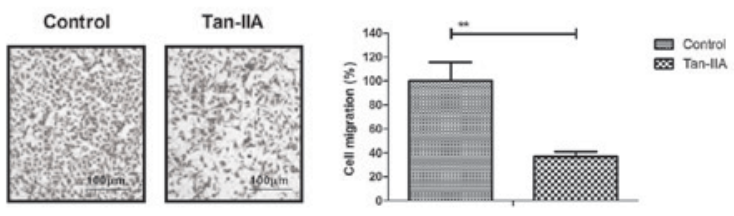

D

C
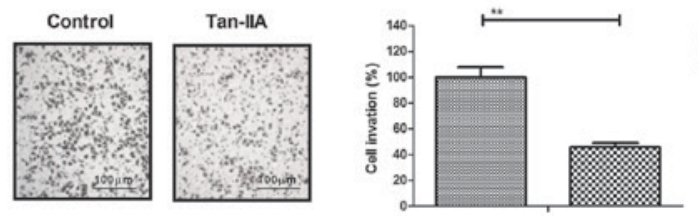

밌

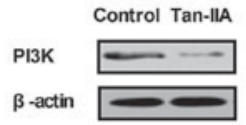

E

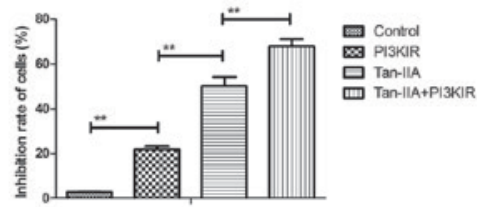

$\mathbf{F}$
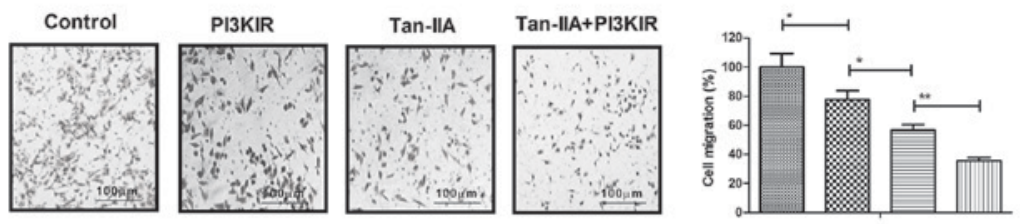

믈 Portrol

Tan-11A

吕 Tan-IA + PIIKKR

G
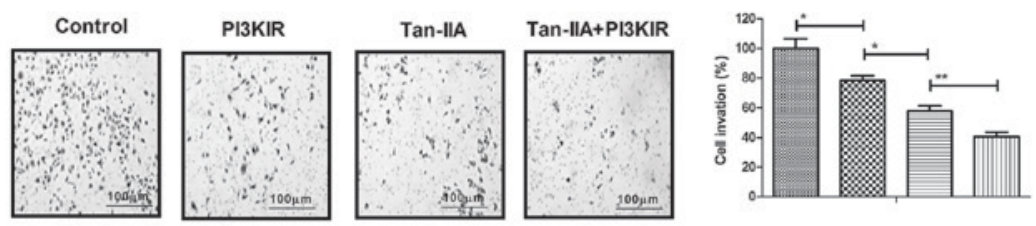

Control

PI3KIR

皿 Tan-\|A

H

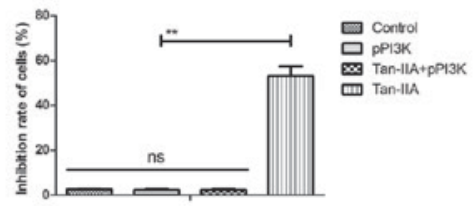

I
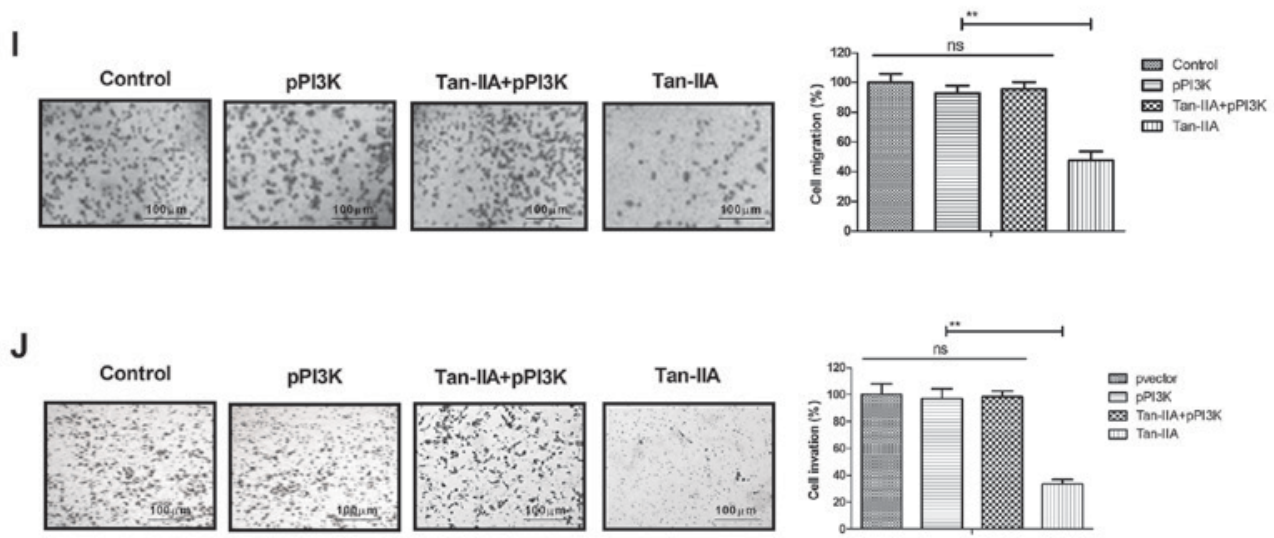

Figure 1. Tan-IIA significantly inhibits ovarian cancer cell growth and aggressiveness through inhibition of target PI3K. (A) Tan-IIA (50, 100, 150, 200 and $250 \mu \mathrm{M})$ inhibited A2780 cell growth. Tan-IIA $(150 \mu \mathrm{M})$ inhibited the (B) migration and (C) invasion of A2780 cells, compared with PBS-treated group. (D) Tan-IIA $(150 \mu \mathrm{M})$ downregulated the expression of PI3K in A2780 cells, compared with the control. PI3K inhibitor (LY294002; PI3KIR) promoted Tan-IIA-inhibited (Tan-IIA+PI3KIR) A2780 cell (E) growth, (F) migration and (G) invasion. Overexpression of PI3K inhibited Tan-IIA-inhibited (H) growth, (I) migration and (J) invasion (J) of A2780 cells. Tan-IIA, tanshinone IIA; PI3K, phosphoinositide 3-kinase; pPI3K, pLentivirus-PI3K; NS, not significant. ${ }^{*} \mathrm{P}<0.05$ and ${ }^{* *} \mathrm{P}<0.01$ vs. control. 
A
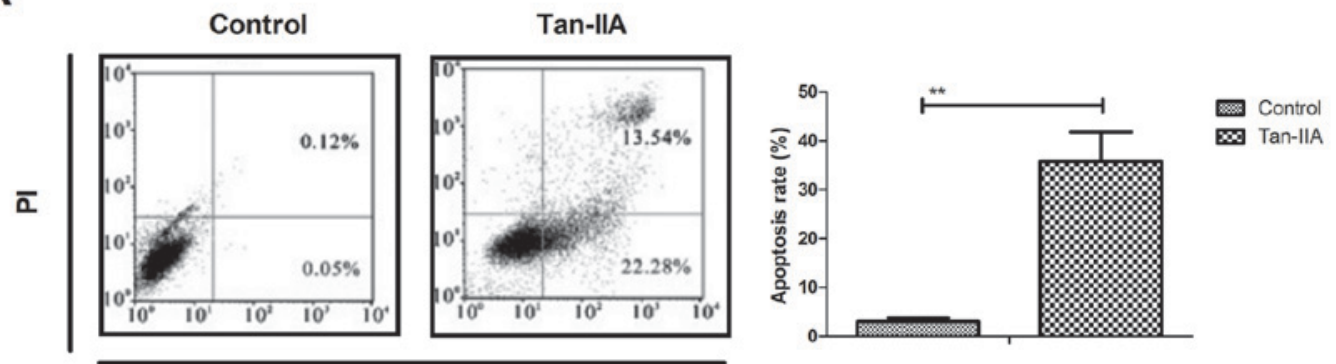

Annexin V-FITC

B

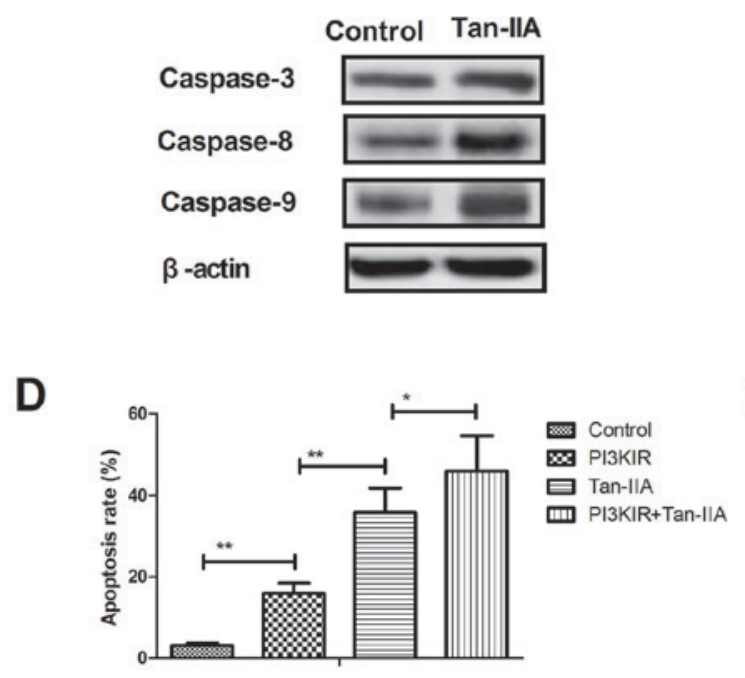

C

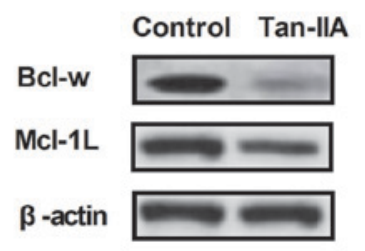

E

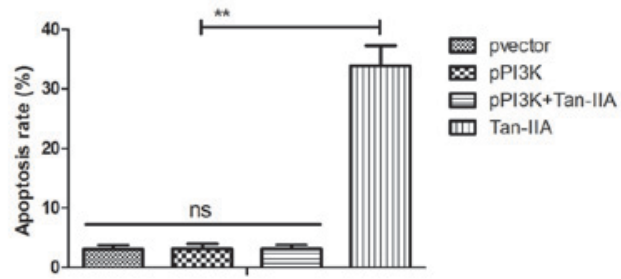

Figure 2. Tan-IIA significantly induces the apoptosis of ovarian cancer cells through inhibition of target PI3K. (A) Tan-IIA significantly increased the apoptosis of human A2780 ovarian cancer cells, compared with the control. (B) Tan-IIA upregulated the expression of caspases-3, caspase-8 and caspases-9 in A2780 cells. (C) Tan-IIA treatment decreased the expression of mitochondrial protective Bcl-w and Mcl-1L in A2780 cells. (D) PI3K inhibitor (LY294002) enhanced Tan-IIA-induced apoptosis of A2780 cells. (E) Overexpression of PI3K eliminated Tan-IIA-induced apoptosis of A2780 cells. Tan-IIA, tanshinone IIA; PI3K, phosphoinositide 3-kinase; Bcl-1, B-cell lymphoma 2-like protein 2; Mcl-1L, myeloid cell leukemia-1 long; pPI3K, pLentivirus-PI3K; ns, not significant. ${ }^{*} \mathrm{P}<0.05$ and ${ }^{* *} \mathrm{P}<0.01$ vs. control.

(3 $\mathrm{min} /$ wash), and then incubated with HRP-conjugated anti-rabbit IgG (Bio-Rad Laboratories, Inc.) at a 1:10,000 dilution for $1 \mathrm{~h}$ at $37^{\circ} \mathrm{C}$. A Ventana Benchmark automated staining system was used for examining the protein expression in tumor tissues.

Terminal deoxynucleotidyl transferase (TdT)-mediated dUTP nick end labeling (TUNEL) analysis. For the analysis of apoptosis of tumor cells in tumor tissues, a TUNEL assay (Biotool, Houston, TX, USA) were used to detect TUNEL-positive cells. The tumor sections were fixed with $4 \%$ paraformaldehyde solution for $60 \mathrm{~min}$ at $4^{\circ} \mathrm{C}$. The cells were then washed with PBS three times and permeabilized by immersing cell slides in $0.2 \%$ TritonX-100 solution in PBS for 30 min at $4{ }^{\circ} \mathrm{C}$. Subsequently, the cells were incubated with equilibration buffer for $30 \mathrm{~min}$ at $4^{\circ} \mathrm{C}$. The myocardial cells were then incubated with $50 \mu \mathrm{l}$ of the reaction mixture at $37^{\circ} \mathrm{C}$ for $60 \mathrm{~min}$, and washed three times with PBS. The cell nuclei were stained with 4',6-diamidino-2-phenylindole for $60 \mathrm{~min}$ at $4^{\circ} \mathrm{C}$. Finally, images of the myocardial cells were captured with a ZEISS LSM 510 confocal microscope (Carl Zeiss AG, Oberkochen, Germany) at $488 \mathrm{~nm}$.
Statistical analysis. All data are expressed as the mean \pm standard deviation of triplicate independent experiments and were analyzed using Student's t-test or one-way analysis of variance with a Tukey's HSD test. All data were analyzed using SPSS 19.0 (IBM SPSS, Armonk, NY, USA) and GraphPad Prism version 5.0 (GraphPad Software, Inc., La Jolla, CA, USA0 with the assistance of Microsoft Excel (Microsoft Corporation, Redmond, WI, USA). $\mathrm{P}<0.05$ was considered to indicate a statistically significant difference.

\section{Results}

Tan-IIA significantly inhibits ovarian cancer cell growth and aggressiveness through the inhibition of target PI3K. The anti-ovarian cancer efficacy of Tan-IIA was analyzed in vitro in the A2780 human ovarian cancer cell line. Following treatment with different concentrations of Tan-IIA (50, 100, 150, 200 and $250 \mu \mathrm{M}$ ) for $24 \mathrm{~h}, \mathrm{~A} 2780$ cell growth was inhibited by the treatment of Tan-IIA (Fig. 1A). The 50\% inhibitory concentration of Tan-IIA for A2780 cells was identified as $150 \mu \mathrm{M}$. As shown in Fig. 1B and C, $150 \mu \mathrm{M}$ of Tan-IIA markedly inhibited the migration and invasion of A2780 cells, compared with that 
A

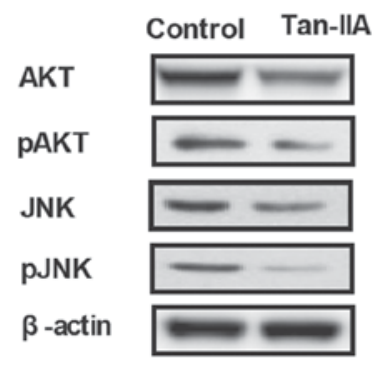

B

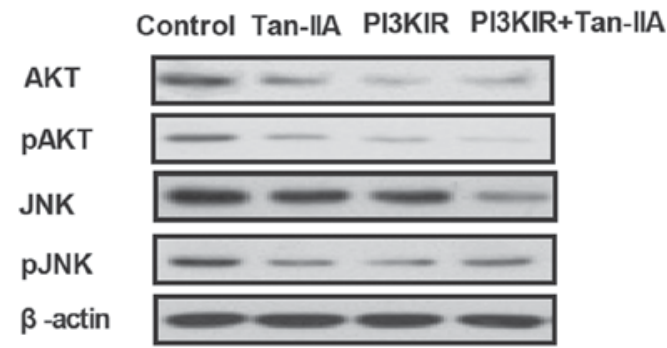

C

pvector pPI3K pPI3K+Tan-IIA Tan-IIA

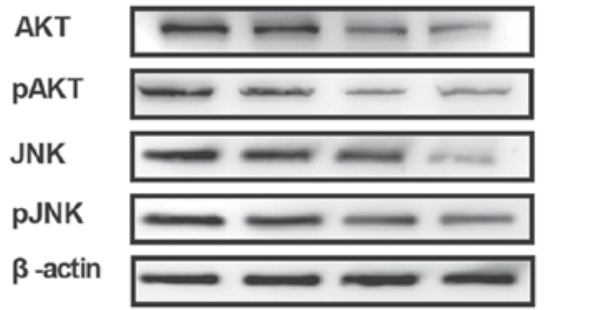

Figure 3. Tan-IIA regulates the expression of key proteins of PI3K/AKT/JNK signaling pathways in ovarian cancer cells. (A) Tan-IIA decreased the expression and phosphorylation of AKT and JNK in A2780 cells. (B) PI3K inhibitor (LY294002) enhanced the Tan-IIA-inhibited expression and phosphorylation of AKT and JNK in A2780 cells. (C) Overexpression of PI3K eliminated the Tan-IIA-inhibited expression and phosphorylation of AKT and JNK in A2780 cells Tan-IIA, tanshinone IIA; PI3K, phosphoinositide 3-kinase; pPI3K, pLentivirus; JNK, c-Jun N-terminal kinase; pJNK, phosphorylated JNK.

in the PBS-treated group (blank control). It was demonstrated that Tan-IIA $(150 \mu \mathrm{M})$ downregulated the expression of PI3K in A2780 cells, compared with that in the control (Fig. 1D). It was also observed that the PI3K inhibitor (LY29400/PI3KIR) enhanced the Tan-IIA-inhibited (Tan-IIA+PI3KIR) growth, migration and invasion of A2780 cells (Fig. 1E-G). However, the overexpression of PI3K eliminated the Tan-IIA-induced inhibited growth and aggressiveness of A2780 cells (Fig. 1H-J). These results indicated that Tan-IIA inhibited ovarian cancer cell growth and aggressiveness through the inhibition of target PI3K.

Tan-IIA significantly induces the apoptosis of ovarian cancer cells through the inhibition of target PI3K. The present study further examined the efficacy of Tan-IIA $(150 \mu \mathrm{M})$ on the apoptosis of ovarian cancer cells. Tan-IIA significantly increased the apoptosis of human A2780 ovarian cancer cells, compared with the control (Fig. 2A). The expression levels of caspases-3, caspase- 8 and caspases- 9 were markedly upregulated by Tan-IIA in the A2780 cells (Fig. 2B). Tan-IIA treatment decreased the expression levels of mitochondria protective $\mathrm{Bcl}-\mathrm{W}$ and Mcl-1L in the human ovarian cancer cells (Fig. 2C). As shown in Fig. 2D and E, the results showed that PI3K inhibitor (LY294002) enhanced the Tan-IIA-induced apoptosis of A2780 cells, whereas the overexpression of PI3K had the reverse effect. These results suggested that Tan-IIA induced the apoptosis of ovarian cancer cells through the inhibition of target PI3K.

Tan-IIA regulates the expression of key protein molecules of the PI3K/AKT/JNK signaling pathways in ovarian cancer cells. To understand the inhibitory effects of Tan-IIA on the apoptosis of A2780 cells, the present study investigated the potential signaling pathway in vitro. Tan-IIA decreased the expression and phosphorylation of AKT and JNK in the A2780 cells (Fig. 3A). In the Tan-IIA-treated group, PI3K inhibitor (LY294002) enhanced the Tan-IIA-induced inhibited expression and phosphorylation of AKT and JNK in the A2780 cells (Fig. 3B). The results indicated that the overexpression of PI3K eliminated the Tan-IIA-inhibited expression and phosphorylation of AKT and JNK (Fig. 3C). These results suggested that Tan-IIA regulated the expression of key protein molecules of the PI3K/AKT/JNK signaling pathways in ovarian cancer cells.

Tan-IIA exerts therapeutic effects on ovarian cancer lesion model rats. The present study further investigated the in vivo therapeutic effects of Tan-IIA on an ovarian cancer lesion model in rats. As shown in Fig. 4A, Tan-IIA treatment significantly inhibited ovarian cancer growth, compared with the PBS-treated rats. The Tan-IIA-treated ovarian cancer lesion model rats showed increased apoptotic bodies in tumor tissues, compared with the PBS-treated rats (Fig. 4B). The immunohistochemical assay demonstrated that the expression levels of PI3K, AKT and JNK were decreased by Tan-IIA treatment in the tumor tissues, compared with those in the control group (Fig. 4C). Tan-IIA treatment significantly prolonged the survival rate of the experimental rats in a100-day observation (Fig. 4D). These results suggested that Tan-IIA exerted therapeutic effects on the rats of the ovarian cancer lesion model.

\section{Discussion}

Ovarian cancer exhibits resistance mechanisms towards chemotherapeutic drugs due to apoptotic resistance (19). A previous study indicated that the attenuation of PI3K and 
A

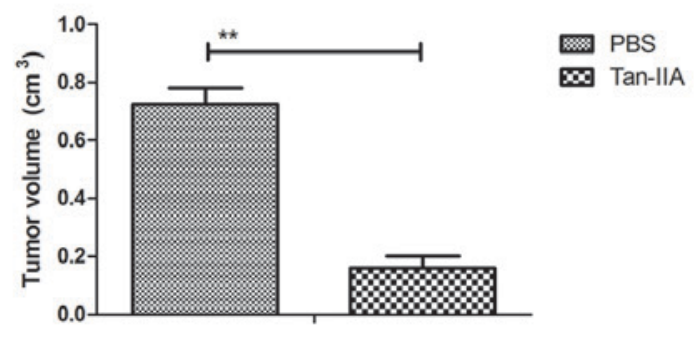

C

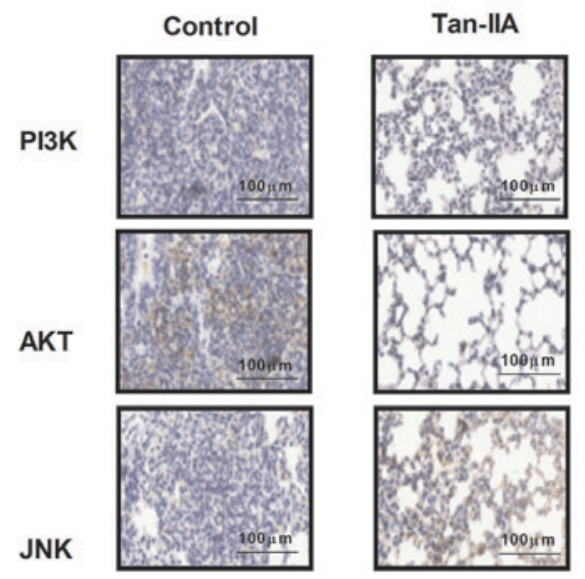

B

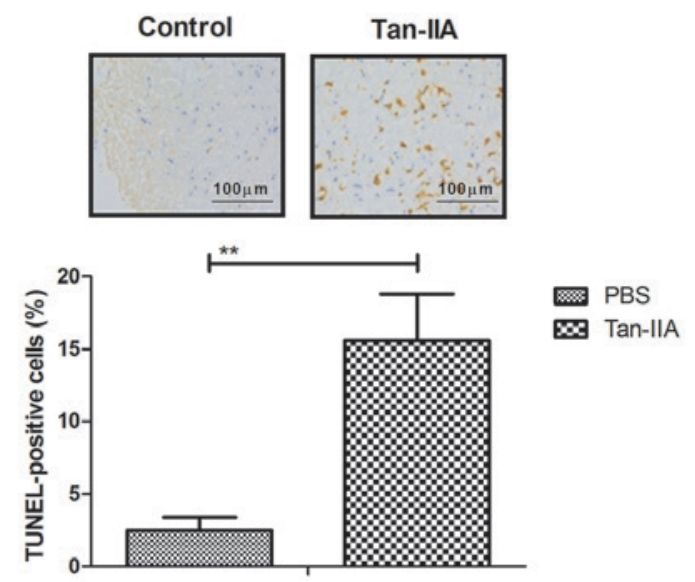

D

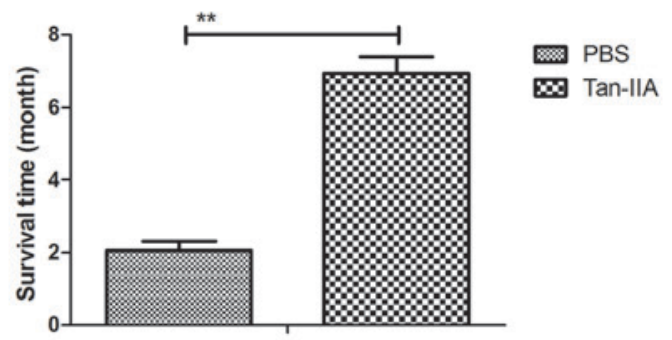

Figure 4. Tan-IIA exerts therapeutic effects in an ovarian cancer lesion model rats. (A) Tan-IIA treatment significantly inhibited ovarian cancer growth, compared with that in PBS-treated rats. (B) Rats in the Tan-IIA-treated ovarian cancer lesion model had increased apoptotic bodies in tumor tissues, compared with the PBS-treated rats. (C) Tan-IIA upregulated the expression levels of PI3K, AKT and JNK in tumor tissues, compared with those in the control. (D) Tan-IIA treatment prolonged survival of experimental rats. Tan-IIA, tanshinone IIA; PI3K, phosphoinositide 3-kinase; JNK, c-Jun N-terminal kinase; PBS, phosphate buffer solution. ${ }^{* *} \mathrm{P}<0.01$ vs. PBS.

extracellular signal-regulated kinase $1 / 2$ contributes to a decrease in the apoptotic resistance of nonadherent ovarian cancer cells (20). Evidence has suggested that Tan-IIA can inhibit ovarian cancer cell growth by inducing apoptosis via the downregulation of p38 MAPK (17). The present study further analyzed the role of Tan-IIA in inducing the apoptosis of ovarian cancer cells in vitro and in vivo. It was found that Tan-IIA significantly inhibited the growth and aggressiveness of ovarian cancer cells via attenuation of the PI3K/AKT/JNK signaling pathways. The findings suggest that Tan-IIA significantly induced the apoptosis of ovarian cancer cells through the upregulation of caspases-3, caspase-8, caspases-9 and the downregulation of Bcl-w and Mcl-1L in A2780 cells.

A previous study demonstrated that Tan-IIA inhibited BxPC3-derived xenograft tumor growth by increasing the protein expression levels of PKR-like endoplasmic reticulum kinase, activating transcription factor 6, inositol-requiring enzyme $1 \alpha$, CCAAT-enhancer-binding protein homologous protein, caspase 3 and caspase 12 (21). The results of the present study showed that Tan-IIA inhibited ovarian tumor growth by inducing apoptosis through the regulation of apoptosis-associated protein expression. Yu et al showed that
Tan-IIA suppressed gastric cancer cell migration by decreasing expression levels of Ki-67, proliferating cell nuclear antigen, matrix metalloproteinase (MMP)-2, MMP-9 and forkhead box M1 (22). In the present study, it was shown that Tan-IIA suppressed the migration and invasion of ovarian cancer cells by inhibiting the target PI3K. Antitumor experiments have indicated that Tan-IIA significantly inhibited growth of Lewis mice with lung cancer by decreasing the expression of Bcl-2 and endostatin (23). The present study demonstrated that Tan-IIA treatment decreased the expression levels of Bcl-w and Mcl-1L in ovarian cancer cells, which contributed to the apoptosis of ovarian cancer cells.

Although the efficacy of Tan-IIA in ovarian cancer has been investigated in a previous study, the molecular mechanism mediated by Tan-IIA has not been clearly documented (17). A previous review revealed that the $\mathrm{PI} 3 \mathrm{~K} / \mathrm{AKT} / \mathrm{mammalian}$ target of rapamycin pathways are potential targeting members in the treatment of ovarian cancer (24). In the present study, the results revealed that Tan-IIA induced the apoptosis of ovarian cancer cells via the downregulation of PI3K/AKT/JNK signaling pathways. Additionally, the in vivo assay showed that Tan-IIA treatment inhibited the growth of ovarian cancer through increasing the apoptosis of tumor cells. In particular, 
the overexpression of PI3K eliminated the Tan-IIA-induced apoptosis of ovarian cancer cells.

In conclusion, the present study provided a novel strategy of Tan-IIA use, which induced ovarian cancer apoptosis by inducing changes in apoptosis-associated genes and causing growth inhibition. The findings of the present study indicated that Tan-IIA offers potential against ovarian cancer through the downregulation of PI3K/AKT/JNK signaling pathways, which indicates the anticancer activity of Tan-IIA for further translation into clinics. Further investigations are required focusing on identifying a novel potential mechanism for the treatment of ovarian cancer.

\section{Acknowledgements}

Not applicable.

\section{Funding}

No funding was received.

\section{Availability of data and materials}

The analyzed data sets generated during the present study are available from the corresponding author on reasonable request.

\section{Authors' contributions}

$\mathrm{XZ}$ and YZ performed the experiments. YEG designed the experiment.

\section{Ethics approval and consent to participate}

This study was approved by the Ethics Committee of the Woman's Hospital, School of Medicine, Zhejiang University (Zhejiang, China).

\section{Patient consent for publication}

Not applicable.

\section{Competing interests}

The authors declare that they have no competing interests.

\section{References}

1. Ahmed-Lecheheb D and Joly F: Ovarian cancer survivors' quality of life: A systematic review. J Cancer Surviv 10: 789-801, 2016.

2. Mohammadi V, Dehghani S,Larijani B and Azadbakht L: Ovarian cancer risk and nonisoflavone flavonoids intake: A systematic review of epidemiological studies. J Res Med Sci 21: 123, 2016.

3. Khoja L, Nolan K, Mekki R, Milani A, Mescallado N, Ashcroft L, Hasan J, Edmondson R, Winter-Roach B, Kitchener HC, et al: Improved survival from ovarian cancer in patients treated in phase III trial active cancer centres in the UK. Clin Oncol (R Coll Radiol) 28: 760-765, 2016.

4. Edwards HM, Noer MC, Sperling CD, Nguyen-Nielsen M, Lundvall L, Christensen IJ and Høgdall C: Survival of ovarian cancer patients in Denmark: Results from the Danish gynaecological cancer group (DGCG) database, 1995-2012. Acta Oncol 55 (Suppl 2): S36-S43, 2016.
5. Tomek S, Horak P, Pribill I, Haller G, Rössler M, Zielinski CC, Pils D and Krainer M: Resistance to TRAIL-induced apoptosis in ovarian cancer cell lines is overcome by co-treatment with cytotoxic drugs. Gynecol Oncol 94: 107-114, 2004.

6. Yang X, Zheng F, Xing H, Gao Q, Wei W, Lu Y, Wang S, Zhou J, $\mathrm{Hu} \mathrm{W}$ and Ma D: Resistance to chemotherapy-induced apoptosis via decreased caspase-3 activity and overexpression of antiapoptotic proteins in ovarian cancer. J Cancer Res Clin Oncol 130: 423-428, 2004

7. Zhong YY, Chen HP, Tan BZ, Yu HH and Huang XS: Triptolide avoids cisplatin resistance and induces apoptosis via the reactive oxygen species/nuclear factor- $\kappa \mathrm{B}$ pathway in SKOV3 ${ }^{\mathrm{PT}}$ platinum-resistant human ovarian cancer cells. Oncol Lett 6: 1084-1092, 2013

8. Lum E, Vigliotti M, Banerjee N, Cutter N, Wrzeszczynski KO, Khan S, Kamalakaran S, Levine DA, Dimitrova N and Lucito R: Loss of DOK2 induces carboplatin resistance in ovarian cancer via suppression of apoptosis. Gynecol Oncol 130: 369-376, 2013.

9. Liang AL, Zhang TT, Zhou N, Wu CY, Lin MH and Liu YJ: MiRNA-10b sponge: An anti-breast cancer study in vitro. Oncol Rep 35: 1950-1958, 2016.

10. Bhargava-Shah A, Foygel K, Devulapally R and Paulmurugan R: Orlistat and antisense-miRNA-loaded PLGA-PEG nanoparticles for enhanced triple negative breast cancer therapy. Nanomedicine (Lond) 11: 235-247, 2016.

11. Su CC and Lin YH: Tanshinone IIA down-regulates the protein expression of ErbB-2 and up-regulates TNF-alpha in colon cancer cells in vitro and in vivo. Int J Mol Med 22: 847-851, 2008.

12. Su CC and Lin YH: Tanshinone IIA inhibits human breast cancer cells through increased Bax to Bcl-xL ratios. Int J Mol Med 22: 357-361, 2008.

13. Wang X, Wei Y, Yuan S, Liu G, Lu Y, Zhang J and Wang W: Potential anticancer activity of tanshinone IIA against human breast cancer. Int J Cancer 116: 799-807, 2005.

14. Lu Q, Zhang P, Zhang X and Chen J: Experimental study of the anti-cancer mechanism of tanshinone IIA against human breast cancer. Int J Mol Med 24: 773-780, 2009.

15. Cheng CY and Su CC: Tanshinone IIA may inhibit the growth of small cell lung cancer H146 cells by up-regulating the Bax/Bcl-2 ratio and decreasing mitochondrial membrane potential. Mol Med Rep 3: 645-650, 2010.

16. Won SH, Lee HJ, Jeong SJ, Lee HJ, Lee EO, Jung DB, Shin JM, Kwon TR, Yun SM, Lee MH, et al: Tanshinone IIA induces mitochondria dependent apoptosis in prostate cancer cells in association with an inhibition of phosphoinositide 3-kinase/AKT pathway. Biol Pharm Bull 33: 1828-1834, 2010.

17. Jiao JW and Wen F: Tanshinone IIA acts via p38 MAPK to induce apoptosis and the down-regulation of ERCC1 and lung-resistance protein in cisplatin-resistant ovarian cancer cells. Oncol Rep 25: 781-788, 2011.

18. Wai-Hoe L, Wing-Seng L, Ismail Z and Lay-Harn G: SDS-PAGE-Based quantitative assay for screening of kidney stone disease. Biol Proced Online 11: 145-160, 2009.

19. Zaffaroni N, Pennati M, Colella G, Perego P, Supino R, Gatti L, Pilotti S, Zunino F and Daidone MG: Expression of the anti-apoptotic gene survivin correlates with taxol resistance in human ovarian cancer. Cell Mol Life Sci 59: 1406-1412, 2002.

20. Tang MK, Zhou HY, Yam JW and Wong AS: c-Met overexpression contributes to the acquired apoptotic resistance of nonadherent ovarian cancer cells through a cross talk mediated by phosphatidylinositol 3-kinase and extracellular signal-regulated kinase 1/2. Neoplasia 12: 128-138, 2010.

21. Chiu TL and Su CC: Tanshinone IIA increases protein expression levels of PERK, ATF6, IRE1 $\alpha$, CHOP, caspase 3 and caspase12 in pancreatic cancer BxPC3 cell-derived xenograft tumors. Mol Med Rep 15: 3259-3263, 2017.

22. Yu J, Wang X, Li Y and Tang B: Tanshinone IIA suppresses gastric cancer cell proliferation and migration by downregulation of FOXM1. Oncol Rep 37: 1394-1400, 2017.

23. Li Q, Hu K, Tang S, Xu LF and Luo YC: Anti-tumor activity of tanshinone IIA in combined with cyclophosphamide against Lewis mice with lung cancer. Asian Pac J Trop Med 9: 1084-1088, 2016.

24. Mazzoletti $\mathrm{M}$ and Broggini M: PI3K/AKT/mTOR inhibitors in ovarian cancer. Curr Med Chem 17: 4433-4447, 2010. 